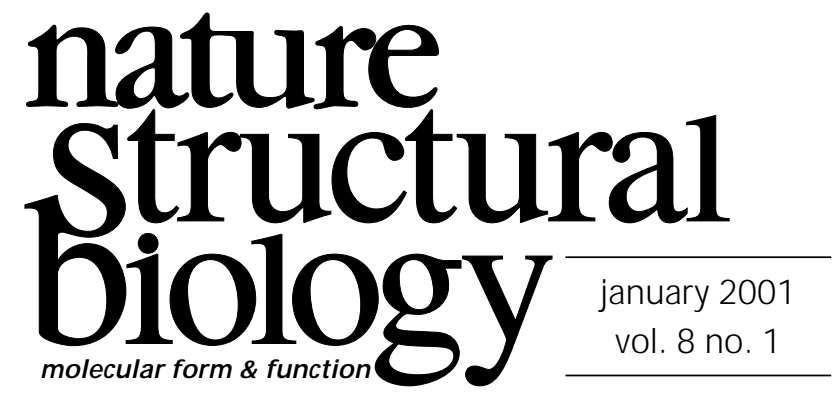

\title{
Understanding spinal muscular atrophy
}

Spinal muscular atrophy (SMA) is a leading cause of death in infancy. It is the second most common autosomal recessive disease (behind cystic fibrosis), occuring in $\sim 1$ in 6,000 live births. In SMA, the anterior horn cells in the spinal cord die, resulting in progressive muscle weakness and ultimately, in some cases, in the inability to breathe and swallow. The gene that is mutated in SMA has been cloned, but we still have little understanding of how the encoded protein - which has been implicated in RNA metabolism - functions to allow the survival of motor neurons. On page 27 of this issue of Nature Structural Biology, Michael Sattler and colleagues present the structure of one domain of this protein, and these results are placed into the context of the cellular machinery by the News and Views on page 13.

There are three main clinical types of SMA, with phenotypes ranging from severe muscular problems in infancy (leading to death before the age of two) to minor muscle weakness in adulthood. In 1995, the telomeric 'survival of motor neuron' (SMN1) gene on human chromosome 5 was cloned ${ }^{1}$. Over $95 \%$ of SMA cases are linked to SMN1; most involve deletions in this gene, but point mutations can also cause the disease. At least two other genes - SMN2 (which is nearly identical to SMN1) and the gene encoding the neuronal apoptosis inhibitory protein (NAIP) affect the severity of SMA.

SMN2 is found near the centromere on human chromosome 5 , sometimes in multiple copies. It differs from SMN1 by only a few bases. One of those base changes affects splicing of the SMN2 transcript, resulting in enhanced exon skipping ${ }^{2}$, which produces a truncated SMN2 protein that cannot fully substitute for SMN1. Mice have the SMN1 gene but not SMN2, and Smn ${ }^{-/-}$ mouse embryos die during implantation in utero. However, if the human SMN2 gene is placed into the $\mathrm{Smn}^{-/-}$mouse genetic background, the mice survive and exhibit an SMA-like phenotype, with changes in the spinal cord and muscle weakness ${ }^{3}$. The NAIP gene is located next to SMN1 and is often deleted along with $S M N 1$ in SMA patients. A loss of function of NAIP may exaggerate SMA severity by allowing increased neuronal apoptosis.

Several lines of evidence implicate the full-length SMN protein in RNA metabolism (specifically, in RNA splicing) ${ }^{4,5}$. Briefly, SMN participates in small nuclear ribonucleoprotein (snRNP) particle assembly in the cytoplasm (likely through its interaction with essential snRNP components called Sm proteins) and may also affect splicing more directly in the nucleus (perhaps by binding to RNA). Several SMN mutations found in SMA patients disrupt $\mathrm{SMN}-\mathrm{Sm}$ interactions ${ }^{6}$, suggesting that the role of SMN in snRNP particle assembly is indeed relevant to the disease, although rigorous proof of this hypothesis is still required. These observations are intriguing because RNA splicing is an essential function in all human cells, and so one might expect additional tissues to be affected in an individual harboring SMN mutations. This could be easily explained if SMN were expressed only in motor neurons, but that is not the case. So, we are left with a perplexing question - how might mutations in SMN lead only to motor neuron death and not to a more widespread phenotype?

Part of the problem in understanding the specificity of such neuronal diseases is that we know rather little about how the various neuronal populations differentiate - about the molecular signals that determine that a given neuron will differentiate into (say) a motor neuron and not a hippocampal neuron. For example, to allow motor neuron differentiation and survival, it may be that a key transcript found only in motor neurons and their precursors must be spliced in a particular way, and this splicing could be regulated by SMN. Research on human pluripotent stem cells is critical for discovering such differences. Thus, it is reassuring that this past August, the National Institutes of Health (after publication of their research guidelines) finally lifted their moratorium on using human pluripotent stem cells in NIH-funded research projects (see http://www.nih.gov/news/stemcell/index.htm). We must hope, and insist, that the next US federal administration (if one is ever finally determined) will not hinder valuable research on stem cells in the coming years, as such work is essential for understanding the molecular pathology of numerous diseases, including SMA.

Unfortunately, at this time little can be done to prevent motor neuron degeneration in SMA patients. However, several molecular strategies for therapeutic intervention might be possible, such as using the SMN protein as a drug, developing small molecules to promote the interaction of SMN and the Sm proteins, or finding a way to decrease exon skipping of SMN2 (thereby allowing it to more fully compensate for the loss of $S M N 1)^{7}$. However, none of these approaches is practical at this time. Gene therapy and treatments with neuronal stem cells are also possibilities for the future, but such treatments are, at best, likely to be many years off, given efficacy, safety, and ethical concerns surrounding both procedures. The new structural information provided by Sattler and colleagues is an important first step toward understanding the details of SMN's molecular interactions, and while their results do not provide all the answers, specific therapies will likely be built on the details that arise from such research.

\footnotetext{
1. Lefebvre, S. Cell 80, 155-165 (1995).

2. Lorson, C.L., Hahnen, E., Androphy, E.J \& \& Wirth, B. Proc. Natl. Acad. Sci. USA 96 6307-6311 (1999)

3. Hsieh-Li, H.M. et al. Nature Genet. 24, 66-70 (2000).

4. Liu, Q., Fischer, U., Wang, F. \& Dreyfuss, G. Cell 90, 1013-1021 (1997).

5. Pellizzoni, L., Kataoka, N., Charroux, B. \& Dreyfuss, G. Cell 95, 615-624 (1998).

6. Pellizzoni, L., Charroux, B. \& Dreyfuss, G. Biochemistry 96, 11167-11172 (1999).

6. Pellizzoni, L., CharrouX, B. \& Dreyfuss, G. Biochemistry 96, 11167-11172 (1999).
7. Hofmann, Y., Lorson, C.L., Stamm, S., Androphy, E.J. \& Wirth, B. Proc. Natl. Acad. Sci. USA 97, 9618-9623 (2000).
} 